where $f: T \rightarrow R^{3}$ is the homeomorphism defined earlier. This $F$ is an imbedding of $S^{0} * T$ in $R^{4}$.

It is clear that there is a mapping $G: R^{4} \rightarrow R^{4}$ such that the associated decomposition space of $R^{4}$ has for its only nondegenerate element the disc $\left\{x \in R^{4}:|x| \leqq 1, x_{3}=x_{4}=0\right\}$. The map $G F \Phi: S^{3} \rightarrow R^{4}$ determines an imbedding of $X^{\prime}$ in 4 -space. Therefore we have supported the claims of the introduction.

Let $K$ be a proper closed subset of $S^{1}$, and for each $t \in K$ let $J_{3}(t)$ $=\left\{z \in S^{3}: z_{1}=t z_{2}\right\}$. Then $\left\{\pi J_{3}(t): t \in K\right\}$ is a family of pairwise linking circles in $R^{3}$. By modifying $\Phi, F$ and $G$ slightly one can imbed the space of this linkage in $R^{4}$.

UNIVERSITY OF VIRGINIA

\title{
A NOTE ON A THEOREM OF LIAO
}

\section{P. E. CONNER}

The purpose of this note is the presentation of a theorem announced by S. D. Liao [3]. After the submission of this note a different proof, by Liao, appeared [4]. We shall state

Theorem 1. Let $\left(S^{1}, S^{n}\right)$ denote the circle group operating as a topological transformation group on the n-sphere. If $M^{r}$ and $N^{n-r-1}$ are two closed, disjoint, invariant submanifolds of $\left(S^{1}, S^{n}\right)$ which have a nonzero linking number, then if $M^{r}$ contains no stationary points, it follows that $N^{n-r-1}$ contains all the stationary points.

Actually, Liao stated the theorem for the action of a cyclic group of prime order, $Z_{p}$, on the sphere. In that case, it is necessary to assume that the invariant submanifolds are linked mod $p$, otherwise the principle of proof is the same. We shall denote the circle group acting on a locally compact, connected, separable metric space $X$ by $\left(S^{1}, X\right)$. We shall denote by $F \subset X$ the set of points in $X$ fixed under the entire group. Throughout this note we shall use AlexanderWallace-Spanier cohomology groups with rational coefficients and closed supports. It is convenient to assume that all spaces are connected. Liao's theorem is an immediate consequence of the following fixed point theorem.

Theorem 2. Let $\left(S^{1}, Y\right)$ and $\left(S^{1}, X\right)$ be two transformation groups and let

$$
f:\left(S^{1}, Y\right) \rightarrow\left(S^{1}, X\right)
$$

Received by the editors December 26, 1958. 
be an equivariant mapping. Suppose there is an integer $m$ such that

(i) $H^{i}(X ; Q)=0$ for $0<i<m$,

(ii) $H^{i}(Y ; Q)=0$ for $i>m$,

(iii) $f^{*}: H^{m}(X ; Q) \rightarrow H^{m}(Y ; Q)$ is nontrivial.

If $Y$ is finite dimensional and $F \subset X$ is nonvoid, then the transformation group $\left(S^{1}, Y\right)$ also has a stationary point.

Before we go on with the proof of this fixed point theorem, let us see how it implies the Liao theorem. The submanifold $M^{r} \subset S^{n}$ is invariant and contains no fixed points by assumption. Let $h^{r} \in H^{r}\left(S^{n}-N^{n-r-1} ; Q\right)$ be dual to the fundamental class of $N^{n-r-1}$. The assumption that $M^{r}$ and $N^{n-r-1}$ are linked is equivalent to the statement that $i^{*}\left(h^{r}\right) \in H^{r}\left(M^{r} ; Q\right)$ is nontrivial, where $i^{*}$ is induced by the inclusion mapping

$$
i: M^{r} \rightarrow S^{n}-N^{n-r-1} .
$$

We note that $H^{i}\left(M^{r} ; Q\right)=0$ for $i>r$. By the Alexander duality theorem, $H^{i}\left(S^{n}-N^{n-r-1} ; Q\right)=0$ for $i<r$. We may apply the fixed point theorem to the transformation groups $\left(S^{1}, M^{r}\right)$ and $\left(S^{1}, S^{n}-N^{n-r-1}\right)$, together with the inclusion map. If $S^{n}-N^{n-r-1}$ contained a fixed point, then $M^{r}$ would also contain a fixed point, which contradicts the assumption of Liao's theorem.

The proof of the fixed point theorem depends on studying the usual spectral sequence associated with a transformation group $[1 ; 2]$. We shall not include details on this point, and we suggest the reader consult the appropriate references. Let $\left(S^{1}, E\right)$ denote the circle group acting freely on a contractable complex $E$. The projection

$$
p: E \rightarrow B\left(S^{1}\right)
$$

of $E$ onto the orbit space $E / S^{1}=B\left(S^{1}\right)$ defines a universal bundle for $S^{1}$. The space $B\left(S^{1}\right)$ is called the classifying space, and its rational cohomology ring $H^{*}\left(B\left(X^{1}\right) ; Q\right)$ is a polynomial ring on a single two dimensional generator. To any transformation group $\left(S^{1}, X\right)$ we associate an auxiliary transformation group $\left(S^{1}, E \times X\right)$ by the diagonal action. This leads to a diagram

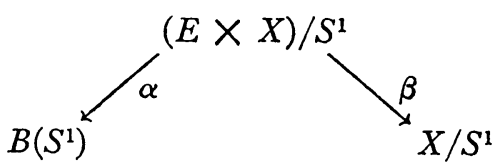

where $\alpha:(E \times X) / S^{1} \rightarrow B\left(S^{1}\right)$ defines a locally trivial fibre bundle with fibre $X$. If the transformation group $\left(S^{1}, X\right)$ has a fixed point, $q$, then the fibre mapping admits a cross-section. In fact, $B\left(S^{1}\right) \times q$ is a subset 
of $(E \times X) / S^{1}$. On the other hand, if $\left(S^{1}, X\right)$ has no fixed points, then by the Vietoris mapping theorem

$$
\beta^{*}: H^{i}\left(X / S^{1} ; Q\right) \cong H^{i}\left((E \times X) / S^{1} ; Q\right) .
$$

Note that $\left(S^{1}, X\right)$ may have finite isotropy groups without having a fixed point.

There is an equivariant mapping

$$
f:\left(S^{1}, Y\right) \rightarrow\left(S^{1}, X\right)
$$

between the two transformation groups which induces a commutative diagram

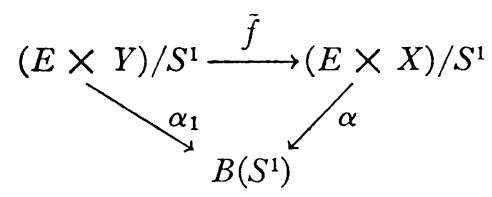

which means $\bar{f}$ is fibre preserving. Let $\left\{E_{r}^{s, t}(Y)\right\}$ and $\left\{E_{r}^{s, t}(X)\right\}$ be the spectral sequences associated with the fibre bundles $\alpha_{1}:(E \times Y) / S^{1}$ $\rightarrow B\left(S^{1}\right)$ and $\alpha:(E \times X) / S^{\prime} \rightarrow B\left(S^{1}\right)$ respectively. Then

$$
\begin{aligned}
& E_{2}^{8, t}(Y) \cong H^{s}\left(B\left(S^{1}\right) ; Q\right) \otimes H^{t}(Y ; Q), \\
& E_{2}^{s, t}(X) \cong H^{8}\left(B\left(S^{1}\right) ; Q\right) \otimes H^{t}(X ; Q),
\end{aligned}
$$

and the $E_{\infty}$-terms are associated with $H^{*}\left((E \times Y) / S^{1} ; Q\right)$ and $H^{*}\left((E \times X) / S^{1} ; Q\right)$. The mapping $\tilde{f}$ induces a homomorphism between spectral sequences; $f_{r}^{*}:\left\{E_{r}^{s, t}(X)\right\} \rightarrow\left\{E_{r}^{s, t}(Y)\right\}$, and $f_{2}^{*}$ has the form

id $\otimes f^{*}: H^{s}\left(B\left(S^{1}\right) ; Q\right) \otimes H^{t}(X ; Q) \rightarrow H^{s}\left(B\left(S^{1}\right) ; Q\right) \otimes H^{t}(Y ; Q)$.

If we assume there is an integer $m$ satisfying the hypothesis of Theorem 2, then we assert that $E_{2}^{0, m}(X) \cong H^{0}\left(B\left(S^{1}\right) ; Q\right) \otimes H^{m}(X ; Q)$ consists entirely of permanent cocycles [1] and furthermore the image of

$($ id $) \otimes f^{*}: H^{s}\left(B\left(S^{1}\right) ; Q\right) \otimes H^{m}(X ; Q) \rightarrow H^{s}\left(B\left(S^{1}\right) ; Q\right) \otimes H^{m}(Y ; Q)$

consists entirely of noncobounding permanent cocycles. Recall that $H^{i}(X ; Q)=0$ for $0<i<m$, so $d_{r}: E_{r}^{0, m}(X) \rightarrow E_{r}^{r, m-r+1}(X)$ must be trivial for $2 \leqq r \leqq m$. To see that $d_{m+1}: E_{m+1}^{0, m}(X) \rightarrow E_{m+1}^{m+1,0}(X)$ is also trivial, we have merely to recall one fact. First of all, the fibre map

$$
\alpha:(E \times X) / S^{1} \rightarrow B\left(S^{1}\right)
$$

admits a cross-section, which insures us that

$$
\alpha^{*}: H^{m+1}\left(B\left(S^{1}\right) ; Q\right) \rightarrow H^{m+1}\left((E \times X) / S^{1} ; Q\right)
$$


is a monomorphism. This in turn means that

$$
d_{m+1}: E_{m+1}^{0, m}(X) \rightarrow E_{m+1}^{m+1,0}(X)
$$

must be trivial [1]. Now, of course, we see that the image

$$
f^{*}: H^{m}(X ; Q) \rightarrow H^{m}(Y ; Q)
$$

consists of noncobounding permanent cocycles in $E_{2}^{0, m}(Y)$. Borel has pointed out, however, that if $f^{*}\left(h^{m}\right) \in H^{m}(Y ; Q)$ is a permanent cocycle, then so is $c^{2 s} \otimes f^{*}\left(h^{m}\right) \in E_{2}^{2 s, m}(Y)$, where $c^{2 s} \in H^{2 s}\left(B\left(S^{1}\right) ; Q\right)$ [2]. Again, a permanent cocycle in $E_{2}^{2 s, m}(Y)$ cannot cobound, since $H^{i}(Y ; Q)=0$ for $i>m$. This implies that $H^{2 s+m}\left((E \times Y) / S^{1} ; Q\right) \neq 0$ for all values of $s$.

At this point we have reached a contradiction, unless $\left(S^{1}, Y\right)$ has a fixed point, for if $\left(S^{1}, Y\right)$ has no fixed point, then

$$
\beta^{*}: H^{28+m}\left(Y / S^{1} ; Q\right) \approx H^{28+m}\left((E \times Y) / S^{1} ; Q\right) \neq 0 .
$$

The contradiction lies in the fact that $Y / S^{1}$ is finite dimensional. (For a proof of the latter statement see [5].)

In closing this note we would like to raise two questions in connection with Theorem 1 . Under the hypothesis of Theorem 1, does $M^{r}$ have to be a rational cohomology sphere? We might drop the assumption that $M^{r}$ contains no fixed points. The set of fixed points $F \subset S^{n}$ is an integral cohomology sphere and a locally orientable generalized manifold. Can it be shown that $M^{r} \cap F$ and $N^{n-r-1} \cap F$ are, in some sense, linked in $F$ ?

Note added in proof: In Theorem 2 it is only necessary to assume $X$ is connected. No such assumption about $Y$ or the fixed point set $F$ is needed.

\section{REFERENCES}

1. A. Borel, Sur la cohomologie des espaces fibres principaux et des espaces homogènes de groupes de Lie compact, Ann. of Math. (2) vol. 57 (1953) pp. 115-207.

2. P. E. Conner, On the action of the circle group, Michigan Math. J. vol. 4 (1957) pp. 241-247.

3. S. D. Liao, Periodic transformations and fixed point theorems, II. Manifolds, Bull. Amer. Math. Soc. Abstract 61-6-845.

4. - Periodic transformations and fixed point theorems. II. Manifolds, Sci. Sinica vol. 7 (1958) pp. 987-1021.

5. D. Montgomery and C. T. Yang, The existence of a slice, Ann. of Math. vol. 65 (1957) pp. 108-116.

UNIVERSITY OF VIRGINIA 